\title{
Acquisition of Cuff-Pulse Waveforms for Estimation of Central Blood Pressure and Additional Hemodynamic Variables
}

\author{
Jiri Jilek \\ Carditech \\ Culver City \\ California \\ USA \\ jjilek30@gmail.com
}

\author{
Milan Stork \\ Department of Applied Electronics and \\ Telecommunications/RICE \\ University of West Bohemia \\ Plzen, Czech Republic \\ stork@kae.zcu.cz
}

\begin{abstract}
Central blood pressure (cBP) is an important indicator of the load on the heart. Because cBP is difficult to measure directly, indirect methods have been developed. We investigated suitability of blood pressure cuff pulse waves for estimation of $\mathbf{c B P}$ and additional hemodynamic variables. Waves obtained from arm cuff and from wrist cuff were compared.
\end{abstract}

Keywords - central blood pressure; cuff-puls;, pulse waves; transfer functio;, systolic pressur;, diastolic pressur; hemodynamic variables

\section{INTRODUCTION}

Investigation of arterial pulse and its contours has a long history. In the second part of 19th century, various types of mechanical sphygmographs were developed. Sphygmographs transformed pulsations in the radial artery into pulse wave recordings. The recordings were considered important for diagnosis of hypertension. After the invention of the blood pressure (BP) cuff sphygmomanometry by Italian physician Riva-Rocci, the interest in the arterial pulse contours declined. Riva-Rocci observed pressure fluctuations in the cuff and called them oscillations. In the late twentieth century, a noninvasive method called applanation tonometry [1] was used by increasing number of researchers interested in pressure waveform contours. The method uses a pencil-shaped tonometer to obtain arterial pressure waveforms. The waveforms were displayed on the computer screen. Skilled application of the tonometer is required to obtain correct waveforms. Most published studies have used waveforms obtained from the radial artery at the wrist. By mathematical manipulation of the waveforms, it was possible to obtain approximation of the central blood pressure (cBP) [2].

Milan Stork's participation was supported by Department of Applied Electronics and Telecommunications, University of West Bohemia, Plzen, Czech Republic and by the Ministry of Education, Youth and Sports of the Czech Republic under the RICE - New Technologies and Concepts for Smart Industrial Systems, project No. LO1607 and the Internal Grant Agency of University of West Bohemia in Plzen, the project SGS-2018-001
In the latter part of the 20th century, arterial pulses in the BP cuff were used by developers of automatic BP monitors. The methods for determination of systolic (SBP), diastolic (DBP), and mean arterial pressure (MAP) were called oscillometric methods or oscillometry [3]. The premise of oscillometry was that the pulses in the cuff were oscillations. The oscillometric methods used amplitudes of cuff-pulses. The contours of the pulses were mostly ignored. In 2010 an article published in the journal Biomedical Instrumentation \& Technology [4] questioned the correctness of oscillometric terminology. The author's claim was that the pulsations in the cuff are arterial pulses, not oscillations. The contours of the pulses recorded at sub-diastolic cuff pressure (CP) were similar to pulse contours recorded by invasive and noninvasive methods. Subsequently, more attention has been paid to arterial pulses obtained from the cuff at subdiastolic pressures. Several instruments originally developed for arterial tonometry started using brachial cuff waves instead of radial artery waves for estimation of cBP. Computer based instruments Sphygmocor Excel [5-6] by Atcor Medical and Vicorder [7] by Skidmore Medical use brachial cuff waves obtained at sub-diastolic $\mathrm{CP}$ for automatic estimation of cBP. We developed an experimental dual-cuff system for noninvasive determination of BP values and additional hemodynamic variables [8]. The dual cuff system uses arterial waves obtained from brachial and wrist cuffs at sub-diastolic CP. The cuffbased automatic methods are better suited for clinical application. Automatic methods for cuff-pulse wave acquisition and utility are assessed in this study. Sphygmocor Excel, Vicorder, and the dual-cuff methods and results are described.

\section{METHODS}

Block diagram of instrumentation necessary for automatic cuff-pulse wave acquisition is in Fig. 1. This type of instrumentation was used in the dual-cuff system [8]. The exact nature of instrumentation used in Sphygmocor Excel and Vicorder is proprietary and

ISBN 978-80-261-0892-4, (C) University of West Bohemia, 2020 
is not disclosed, but it is similar to the instrumentation described below.

The computer controls all necessary procedures via USB interface and instrumentation module. The module provides digital controls, analog-to-digital conversion (ADC) and USB interface. Proprietary software is used for this task. The air pump provides cuff inflation procedures and the air valve controls cuff deflation and CP hold. Pressure transducer, amplifier, and filter provide analog signal for analogto-digital converter (ADC). The filter is high pass filter with cutoff frequency $f_{\mathrm{c}}=0.5 \mathrm{~Hz}$. The $0.5 \mathrm{~Hz}$ cutoff frequency is sufficient for removal of the $\mathrm{CP}$ descending ramp and for preservation of the low frequency components of cuff pulse waveforms. Higher cutoff frequencies can result in cuff wave distortion, especially at low heart rates. At low heart rate and high cutoff frequency the diastolic segment of the waveform becomes depressed.

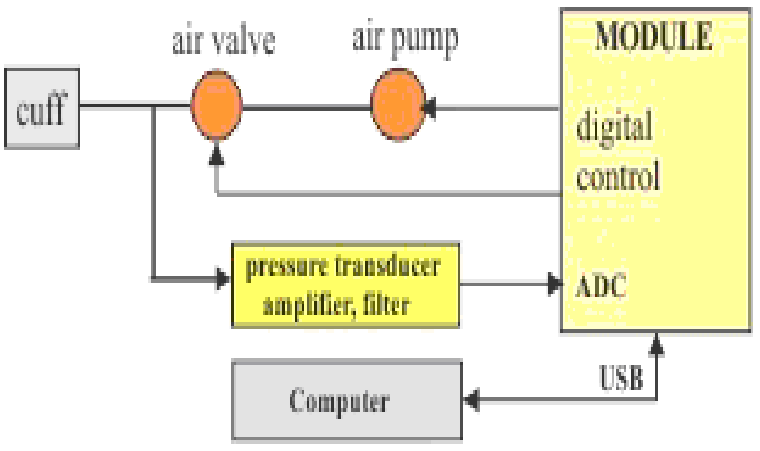

Figure 1. Instrumentation for cuff-pulse acquisition includes cuff, air valve, air pump, pressure transducer, amplifier, filter, analog-todigital converter (ADC),USB interface, and computer

The waves acquired from the cuff at sub-diastolic CP do not provide BP values and must be calibrated. The calibration values are provided by automatic SBP and DBP determination. Cuff-pulse wave amplitudes and $\mathrm{CP}$ values are usually used for this task. Cuff waves acquired during gradual cuff deflation and cuff waves acquired at CP just below DBP are shown in Fig. 2.

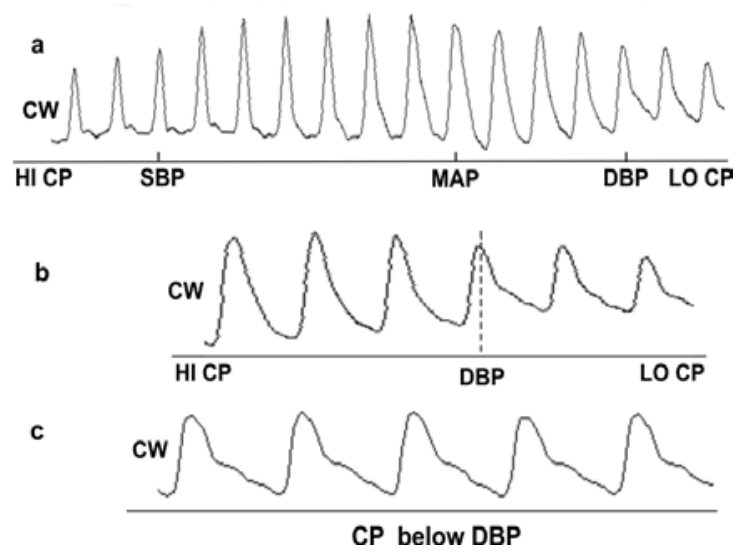

Figure 2. A Cuff-waves (CW) during gradual cuff deflation, bcuff-waves near DBP point, c- cuff-waves at CP below DBP

The algorithm for cuff-wave acquisition and calibration for $\mathrm{cBP}$ :
1. Values of SBP and DBP are algorithmically determined from $\mathrm{CP}$ values and cuff-wave $(\mathrm{CW})$ amplitudes (Fig 2 a).

2. The computed DBP value is used for the CP level for wave acquisition. $\mathrm{CP}$ is held at sub-diastolic pressure and cuff-waves are acquired (Fig $2 \mathrm{c}$ ). The sample size is usually about 5-10 seconds.

3. The acquired cuff-waves are assigned calibration values of SBP and DBP.

4. Transfer function is applied to the calibrated waves and central BP values are computed. Transfer functions are proprietary.

The determination of SBP and DBP values is usually based on the determination of MAP. MAP is obtained by finding the largest amplitude of cuff waves (Fig 2 a). Characteristic ratio method was first introduced by Geddes [10]. According to Geddes SBP corresponds to $50 \%$ of maximal cuff-pulse amplitude on the upslope of amplitude envelope. DBP corresponds to $80 \%$ of maximal amplitude on the down slope of amplitude envelope. Another method claims that SBP is at $40 \%$ and DBP at $85 \%$ of maximal amplitude. The characteristic ratio methods are based on statistical estimates, not on physiological principles. Commercial BP monitors use undisclosed methods. No standard method is in existence. Methodological differences result in different BP values. These differences affect accuracy of cBP estimation. Computed DBP values serve as reference points for cuff-wave acquisition. Sphygmocor Excel uses CP equal to DBP- $10 \mathrm{mmHg}$ for cuff-wave acquisition. Vicorder uses a fixed value of $70 \mathrm{mmHg}$. Fig. 3 shows Sphygmocor brachial cuff waves acquired at $\mathrm{CP}=\mathrm{DBP}-10 \mathrm{mmHg}$.

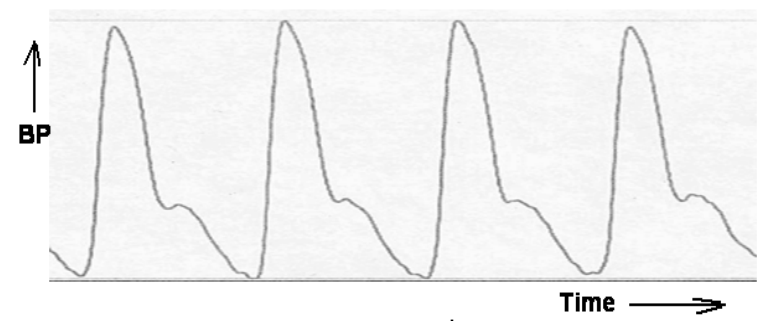

Figure 3. Sphygmocor Excel brachial cuff waves acquired at $\mathrm{CP}=$ DBP- $10 \mathrm{mmHg}$

Cuff-wave acquisition for determination of stroke volume (SV) and derived variables are similar to the algorithm for cBP. The difference is that the acquired waves do not need to be calibrated and transfer function is not applied. Instead, left ventricular ejection time (LVET), SV, cardiac output (CO), total peripheral resistance (TPR), and systemic arterial compliance (SAC) are computed. A study using arm cuff waveforms and estimated BP values obtained with an experimental dual-cuff system showed that hemodynamic variables obtained from the cuff can be successfully estimated [9]. The system used cuff waveforms obtained at CP just below DBP value. 


\section{RESUlTS}

The study by Pucci et al [6] evaluated the Vicorder instrument and compared it to the Sphygmocor Excel cuff-based device. Central SBP obtained with each device satisfied the American Association for the Advancement of Medical Instrumentation accuracy criteria when cuff waveforms were calibrated to invasive MAP. Estimated - invasive cSBP mean difference was $-4.0 \mathrm{mmHg}$. When brachial cuff SBP/DBP was used for calibration cSBP was underestimated by $-6.4 \mathrm{mmHg}$. Sphygmocor cSBP was underestimated by $-11.9 \mathrm{mmHg}$ when invasive MAP was used.

Peng et al [7] compared Sphygmocor Excel cuffbased device to Sphygmocor ToncBP tonometric device. The mean difference SD for central SBP, central DBP, and central pulse pressure was - $0.89 \pm 3$ $\mathrm{mmHg}$. The conclusion was that Sphygmocor Excel and Sphygmocor ToncBP were substantially equivalent.

The dual-cuff system study compared hemodynamic variable values computed from brachial cuff waves to values obtained from wrist cuff waves. The brachial cuff and wrist cuff waves were acquired simultaneously. Mean values of brachial stroke volume (SV) were $83 \mathrm{ml}$ and cardiac output $(\mathrm{CO})=6$ $1 / \mathrm{min}$. Wrist cuff derived $\mathrm{SV}=81 \mathrm{ml}$ and $\mathrm{CO}=5.8$ $1 / \mathrm{min}$.

\section{DISCUSSION}

The automatic cuff-based methods and results described in this paper showed that it is feasible to utilize pressures and waves from the brachial cuff to obtain hemodynamic variables that are similar to those obtained by other direct and indirect methods. Values of cBP have been usually obtained by arterial tonometry from radial artery. Skilled application of the tonometric probe is required and automatic operation is not possible with arterial tonometry. On the other hand, radial artery tonometry can provide more accurate waves that are used to reconstruct aortic pressure wave and compute cBP. Brachial cuff derived waves are dampened by large volume of air in the cuff [7] thus decreasing overall accuracy. Underestimation of cBP by Sphygmocor and Vicorder could be caused by wave dampening and also by errors in estimated calibration values of SBP and DBP. Another potential accuracy problem is the CP level at which the cuff waves are acquired. Sphygmocor Excel acquires the waves at DBP- $10 \mathrm{mmHg}$. This can be a problem where the values of DBP are low. Wave amplitudes decrease quickly from the point of MAP to the end of cuff deflation. This can be seen in Fig. 2 a. Acquiring waves at DBP- $10 \mathrm{mmHg}$ level can result in low wave amplitudes. Low amplitude waves are more susceptible to artifacts, which in turn leads to decreased accuracy. The problem with Vicorder is that it acquires cuff waves at fixed $\mathrm{CP}$ of $70 \mathrm{mmHg}$. At low DBP values the $70 \mathrm{mmHg}$ acquisition level is too high and leads to wave distortion and deceased accuracy. This can be seen in Fig. 2 b. For example, when the measured DBP is $60 \mathrm{mmHg}$, the $70 \mathrm{mmHg}$ acquisition level is too high and the acquired waves will be distorted. In hypertension, the DBP values are frequently in excess of $90 \mathrm{mmHg}$. In such cases the waves acquired at $70 \mathrm{mmHg}$ may have low amplitudes. For the above reasons, the optimal CP level for wave acquisition is at or just under DBP level.

Brachial cuff method was chosen for Sphygmocor Excel and Vicorder because it provides a convenient, user friendly, automatic method. The disadvantage is lower quality waves and larger errors in cBP estimation. Potentially more accurate method is utilization of cuff-waves from wrist cuff. Wrist cuff and brachial cuff waves recorded simultaneously are shown in Fig. 4. The waves were acquired by the dualcuff system.

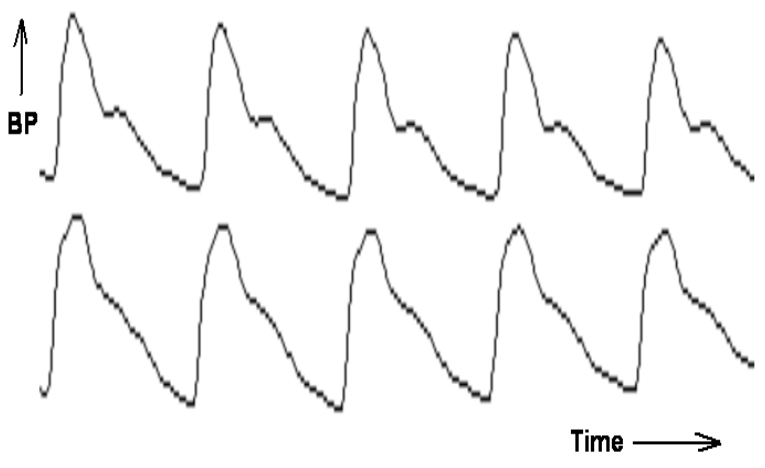

Figure 4. Wrist cuff waves (top trace) and brachial cuff (bottom trace) acquired simultaneously

Wrist cuff waves are more accurate because the volume of air in the cuff is much smaller than the volume in the brachial cuff and there is less dampening. Another factor contributing to wave dampening is the amount of soft tissue under the cuff. The amount of soft tissue under the wrist cuff is usually much smaller, especially in obese patients. The wrist cuff waves in Fig. 4 have better defined contours than the waves from the brachial cuff. Acquisition and comparison of wrist cuff waves to the waves acquired by radial artery tonometry was described previously [11].

The dual cuff system has several advantages over Sphygmocor Excel and Vicorder:

1. The dual cuff system uses a brachial and a wrist cuff for increased accuracy of BP determination [12]. Sphygmocor Excel and Vicorder use commercial BP monitors of unknown accuracy.

2. Cuff waves acquired from wrist cuff are more accurate than waves from brachial cuff.

3. The dual cuff system uses DBP level for acquisition of waves. Better signal to noise ratio can be achieved.

The dual cuff system uses 2 separate pneumatic and analog circuits for each cuff. The block diagram in Fig. 1 shows only the circuits for brachial cuff because a system for $\mathrm{cBP}$ determination uses only 1 cuff. The dual-cuff system has been used mainly for improved accuracy of BP determination and for estimation of hemodynamic variables. At this time the dual cuff system does not include cBP estimation. 


\section{CONCLUSION AND FUTURE WORK}

Acquisition and utilization of arterial waves from the BP cuff represents important step forward. Automatic operation of the systems described above makes such instruments suitable for clinical applications. Automatic BP monitors can add additional hemodynamic variables to their functions. Even home BP monitors can be equipped with such functions and telemonitoring [13] will make sophisticated patient management part of telemedicine. Improved accuracy and reliability of cuff-pulse derived functions is important. Improved accuracy could involve utilization of multiple cuffs [14] while fully automatic operation can still be accomplished

\section{REFERENCES}

[1] R. Kelly, C. Hayward, A. Avolio, M. O'Rourke, "Noninvasive Determination of Age Related Changes in the Human Arterial Pulse," Circulation 1998; 80:1652-1659.

[2] J. D. Cameron, B. McGrath, A. M. Dart; "Use of Radial Artery Applanetion Tonometry and a Generalized Transfer Function to Determine Aortic Pressure Augmentation in Subjects With Treated Hypertension," J Am Coll Cardiol 1998; 32:1214-1220.

[3] J. Jilek, T. Fukushima, "Oscillometric Blood Pressure Measurement: The Methodology, Some Observations, and Suggestions," Biomed Instrum \& Technol 2005;39:237-241.

[4] J. Jilek, "Oscillations or Cuff-Arterial Pulses?" Biomed Instrum \& Technol 2010; 44:6.
[5] X. Peng, M. G. Schultz, W. P. Abhayratna, M. Stowasser, J. E.Sharman, "Comparison of Central Blood Pressure Estimated by a Cuff-based Device with Radial Tonometry,". Am J Hypertens 2016; 29:1173-1178.

[6] X. Peng, M. G. Schultz, D. S. Picone, N. Dwyer, J. A. Black, P. Roberts-Thomson et al., "Non-invasive measurement of reservoir pressure parameters from brachial-cuff blood pressure waveforms," J Clin Hypertens 2018;20:1705-1711.

[7] G. Pucci, J. Chriyan, A. Hubsch, S. S. Hickson, P. R. Gajendragkadkar, T. Watson et al., "Evaluation of the Vicorder, a novel cuff-based device for the nonivasiveestimation of central blood pressure," J Hypertens 2013; 31:77-85.

[8] .J. Jilek, M. Stork, "Wrist Cuff Emulates Auscultation in Dua-Cuff in Noninvasive Blood Pressure System," Proc of Int Conf Applied Electronics, Plzen, Czech Republic, 2012.

[9] J. Jilek, M. Stork, "Estimation of Hemodynamics From Arm Blood Pressure Cuff Waveforms," Proc of Int Conf Applied Electronics, Plzen, Czech Republic, 2013

[10] L. A. Geddes, "Characterization of the oscillometric method for measuring indirect blood pressure.," Ann Biomed Eng 1982;16:271-280

[11] J. Jilek, M. Stork, "A wrist cuff method for acquisition and analysis of radial artery waveforms," Proc of Int Conf Applied Electronics, Plzen, Czech Republic, 2007.

[12] J. Jilek, M. Stork, "Determination of systolic, mean and diastolic blood pressures with dual cuff system is based on physiology,".AE2016 International Conference Proceedings, Plzen, Czech Republic., 2016.

[13] G. Parati, E. Dolan, R. J. McManus, S. Omboni,"Home blood pressure telemonitoring in the 21st century," J Clin Hypertens 2018;20:1128-1133.

[14] J. Jilek, M. Stork, "Assessment of Three Multiple Cuff Blood Pressure Devices," AE2019 International Conference Proceedings, Plzen, Czech Republic, 2019. 